\title{
BEAM EXTRACTION CONCEPTS AND DESIGN FOR THE UNIVERSITY OF MARYLAND ELECTRON RING (UMER)*
}

\author{
M. Walter", T. Godlove, G. Bai, B. Beaudoin, S. Bernal, D. Feldman, I. Haber, R.Kishek, \\ P. O'Shea, C. Papadopoulos, M. Reiser, D. Stratakis, D.Sutter, J.C.T. Thangaraj, C. Wu \\ IREAP, University Maryland, College Park, MD 20742 U.S.A.
}

\begin{abstract}
The University of Maryland Electron Ring (UMER) is a low energy, high current recirculator for beam physics research. The electron storage ring has been closed and recent operations have been focused on achieving multiturn transport. An entire suite of terminal diagnostics is available for time-resolved phase space measurements of the beam. These diagnostics have been mounted and tested at several points on the ring before it was closed and will complete the ring when mounted to the extraction section. UMER utilizes a unique injection scheme which uses the fringe fields of an offset quadrupole to assist a pulsed dipole in bending the beam into the ring. Similar concepts, along with a more traditional electrostatic method, are being considered for beam extraction. This presentation will focus on the recent efforts to design and deploy these major subsystems required for beam extraction.
\end{abstract}

\section{INTRODUCTION}

The University of Maryland Electron Ring (UMER) is a low energy, high current recirculator for beam physics research [1]. UMER is an extremely versatile experimental platform. The beam current pulse may be adjusted in discrete steps from $0.6 \mathrm{~mA}$ to $100 \mathrm{~mA}$, while the pulse length is as long as $100 \mathrm{~ns}$. The beam current profiles are adjustable in both space and time. UMER is addressing issues in beam physics relevant to many applications that require intense beams of high quality, such as advanced concept accelerators, free electron lasers, spallation neutron sources, and future heavy-ion drivers for inertial fusion.

The electron ring has recently been closed and experimental efforts have been largely focused on achieving multi-turn operation [2,3]. With the current experimental setup terminal diagnostics (beam envelope measurements, energy spread, etc.) may be used on subsingle-turn transport, however, if longer transport lengths are desired only non-terminal diagnostics (beam position monitors, current measurement, etc.) may be used. The last major subsystem required on the ring is the extraction line, which will allow a full set of terminal beam diagnostics to be applied on a turn by turn basis. The primary focus of this presentation is to show the preliminary design concepts for extraction of the UMER electron beam.

A brief description of injection in UMER will aid in the understanding of the proposed extraction schemes.

*This work is supported by the US Dept. of Energy grant numbers DEFG02-94ER40855 and DE-FG02-92ER54178, and by the Office of Naval Research and the Joint Technology Office.

\#mwalter@umd.edu

01 Circular Colliders
UMER uses a unique scheme for injection which incorporates the dipole moment of an offset quadrupole in combination with a pulsed dipole to provide the $10^{\circ}$ bend required [4]. A short steering dipole is used to control the beam's position as it enters the offset quadrupole. The pulsed dipole must be operated in opposite polarities for the injected beam and the recirculating beam, and must achieve the polarity change in the interval between when the tail of the injected beam exits the dipole and the head of the recirculating beam arrives $(\sim 100 \mathrm{~ns})$. The polarity of the entire ring is determined by the offset quadrupole because it must be defocusing in the plane of the ring for it to contribute to the left bend (as seen by the electrons) on injection and right bend on recirculation.

Three major design candidates for beam extraction have been developed. The first to be discussed in this presentation is a pulsed magnetic dipole in combination with an offset quadrupole used to extract the beam in the horizontal plane of the ring. The second concept utilizes a similar pulsed magnetic dipole but extracts the beam vertically. Both of these pulsed magnetic schemes are similar to the inverse of the system used for injection in UMER. The third candidate, an electrostatic extraction design, will also be described.

\section{EXTRACTION SCHEMES}

\section{Magnetic Horizontal Extraction}

It is important to note that extraction of the beam in the horizontal direction cannot be achieved by simply flipping a copy of the injection $\mathrm{Y}$ because the sense (focusing or defocusing) of the ring quadrupoles is fixed by injection such that the quadrupole prior to every dipole is defocusing in the plane of the ring. In order to use a quadrupole to aid in the bending for extraction, the extraction point must be located in the straight section prior to the defocusing quadrupole. Figure 1 shows the top view of this scheme, where YQ is the defocusing offset quadrupole and PD is the pulsed dipole added to

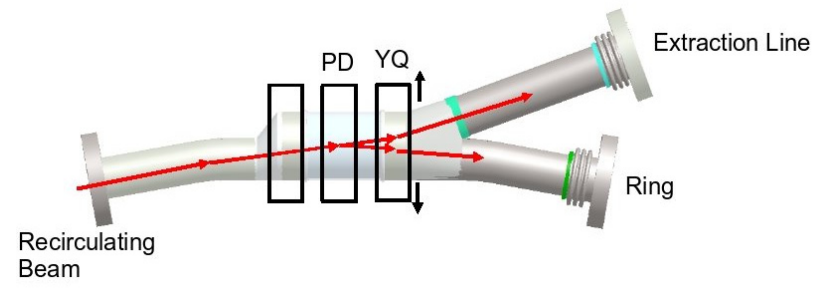

Figure 1: Horizontal extraction using the combined effect of a fast pulsed dipole (PD) and offset quadrupole (YQ) to achieve the net bend angle required.

T12 Beam Injection/Extraction and Transport 
the straight drift section. A short section of glass $(\sim 10$ $\mathrm{cm})$ must be positioned under the pulsed dipole to allow the fast pulsed magnetic field to stabilize as the beam passes through this section. A slightly resistive coating of $\mathrm{Al} / \mathrm{AlO} 2$ is deposited on the inner wall of the glass which prevents any possible charge buildup on part of the vacuum boundary pipe.

The recirculating beam enters the extraction section from the left and either gets a right bend from the pulsed dipole to continue in the ring, or a left bend to send it down the extraction line. YQ aids in the bending in both directions because it is defocusing in the horizontal plane and therefore reduces the bending demanded of the pulsed dipole. A small dipole kicker must be added to each arm after YQ to get the beam back on the proper trajectory for recirculation or straight down the centerline for extraction.

The fast current pulse required for magnetic extraction is shown in Figure 2. The fast $(\sim 1 \mu \mathrm{s})$ current pulse is superimposed on the long recirculating current pulse. Similar to our injection scheme, the extraction pulse must reach its design value before the head of the beam arrives after its last lap of the ring. The fast extraction pulse must also hold constant while the beam passes through the magnet, approximately $100 \mathrm{~ns}$ or slightly longer if we allow the beam to expand longitudinally. The recirculating pulse must be long enough to allow the beam at least 100 turns of the ring, or approximately $20 \mu$ s.

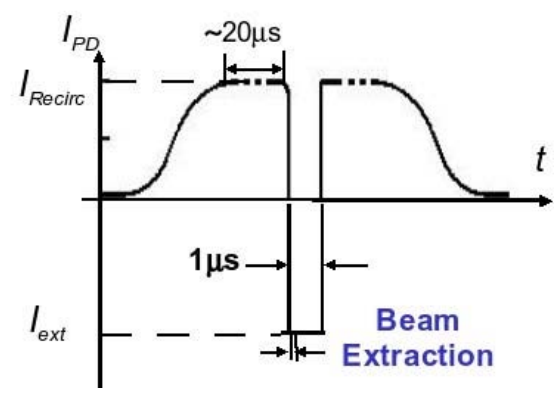

Figure 2: Timing schematic for the pulsed elements for magnetic horizontal extraction.

\section{Magnetic Vertical Extraction}

Vertical extraction of the beam via a pulsed dipole is similar to the horizontal case just described. Figure 3 shows the side view of a possible vertical extraction setup. Once again we have the combined effort of a fast pulsed dipole (PD) and offset quadrupole (YQ) to achieve

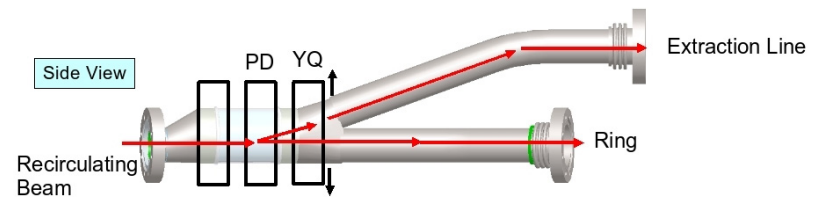

Figure 3: Vertical extraction using the combined effect of a fast pulsed dipole (PD) and offset quadrupole (YQ). the desired bend angle for extraction. The difference is that now the dipole bends the beam vertically for extraction and can therefore be co-located with the normal bending dipole of the ring. YQ is defocusing in the vertical direction and aids in the bending because it takes the place of a normal ring quadrupole just downstream of the dipole.

The recirculating beam would enter the extraction section from the left and get only the normal $10^{\circ}$ horizontal bend for recirculation from the ring dipole leaving the pulsed dipole off, or a vertical bend from the pulsed dipole for extraction. YQ would be located with its magnetic center in the ring plane so that it would only do additional bending when the beam moves above the recirculating path. Once again a short section of glass with a resistive wall coating must be placed under the pulsed dipole to allow for magnetic field penetration. One additional dipole would be added to the extraction line so that the extracted beam could be brought back to a horizontal trajectory slightly above the plane of the ring.

The current pulse required for this extraction design would be similar to that shown in Fig. 2, although the recirculating current pulse would be zeroed because the ring dipole would take care of recirculation bending in the horizontal plane.

\section{Electrostatic Horizontal Extraction}

The third option examined for extraction of the beam utilizes a fast pulsed electric field to bend the beam. Figure 4 shows the top view of a possible electrostatic extraction setup. In order to use this extraction scheme one of the regular ring dipoles must be replaced by two vertical plates across which an electric field is induced. A radial electric field (relative to the ring center) would then be applied to the beam for recirculation and reversed for beam extraction.

The current pulse required for this extraction design would be similar to that shown in Fig. 2, except that the current switching would be replaced by high voltage switching on the vertical axis of the graph.

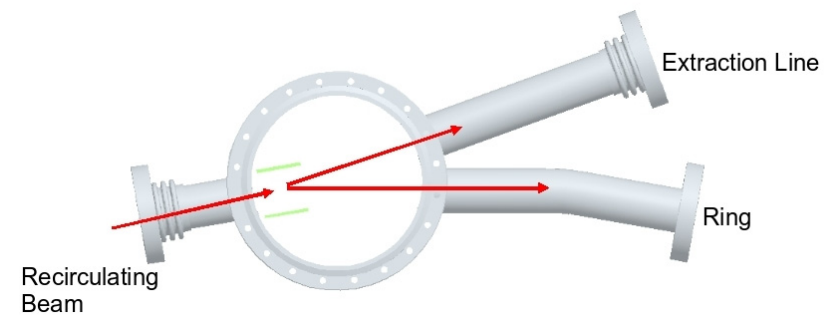

Figure 4: Horizontal extraction using electrostatic bending.

\section{DISCUSSION}

Each of the three cases presented for beam extraction has its own set of advantages and difficulties. Many lessons have been learned from the pulsed magnetic injection method used in UMER which may also be applied to beam extraction. Both of the fast pulsed 
magnetic schemes place extreme demands on the current pulsing circuit. Experience now shows that the current required to bend the beam $10^{\circ}$ for recirculation is on the order of $10 \mathrm{~A}$, while the $10^{\circ}$ bend needed for injection requires nearly $30 \mathrm{~A}$ because it opposes the earth's magnetic field. The net current change is then $40 \mathrm{~A}$ which must occur in 100ns or less. Fast switching of such high current values into an inductive load is difficult but possible with recent upgrades to the circuit [5]. The fall time of the fast pulse is critical for injection, however this will be reversed for extraction where the rise time must be fast enough to change the dipole field between the time that the tail of the beam exits the section on its last turn of the ring and stabilize before the head of the beam arrives for extraction. Ringing in the circuit which causes steering problems for injection and recirculation will not be of such great importance in extraction since extraction involves only a single pass of the beam.

The horizontal magnetic extraction scheme has the advantage that it is the closest design to what is currently used for injection in UMER. One possible disadvantage of this scheme is that it perurbs the recirculating orbit of the beam on each pass through the extraction section, although initial calculations indicate that the perturbation is likely to be negligible. The vertical magnetic extraction scheme eliminates this perturbation to the recirculating beam and allows us to position the pulsed dipole at a regular ring dipole location. Construction of the vertical magnetic extraction section will be slighly more complex due to addition of the vertical dimension of alignment and support. The electrostatic extraction scheme has the primary advantage of voltage switching over current switching in the magnetic designs, however care must be taken in this case to keep capacitances at a minimum. The electrostatic scheme will also require a custom chamber to be built which will contain at least one focusing quadrupole of the ring. Current design of the quadrupoles utilizes a dual layer printed circuit which is not vacuum compatible.

Another lesson learned from injection studies on UMER is the need for beam position monitoring at critical locations near the offset quadrupole and pulsed dipole. The final design of the extraction section will incorporate beam position monitors (BPM) within the vacuum boundary pipe adjacent to PD and YQ to allow precision control of the beam through each step of extraction.

\section{CONCLUSIONS}

Three designs have been forwarded for consideration for beam extraction on UMER. The three designs are magnetic horizontal extraction, magnetic vertical extraction, and electrostatic horizontal extraction. One design will chosen after careful consideration of each design's advantages. That design will then be advanced to construction and installation in the near future.

\section{REFERENCES}

[1] M. Reiser et al., "The Maryland Electron Ring for Investigating Space-Charge Dominated Beams in a Circular FODO System," Proc. 1999 Part. Accel. Conf., New York, IEEE, 1999, p. 234.

[2] S. Bernal et al., "New Developments In SpaceCharge Beam Physics Research at the University Of Maryland Electron Ring (UMER)," Proc. 12th Workshop on Advanced Accelerator Concepts (AAC), Lake Geneva, WI, 10-15 July, 2006, ed., M. Conde and C. Eyberger, (New York: AIP Press 877, 2006), p. 94.

[3] M. Walter et al., "Commissioning of the University of Maryland Electron Ring (UMER): Advances toward multi-turn operation," Physics of Plasmas 13, 056703 (2006).

[4] H. Li et al., "Beam Control and Matching for the Transport of Intense Beams," Nuc. Inst. and Meth. A, 544, (2005), pp 367-373 .

[5] M. Holloway et al., "Injector Electronics for Multiturn Operation of the University of Maryland Electron Ring (UMER)," Proc. 2005 IEEE Part. Accel. Conf., Knoxville, TN, ed. C. Horak, IEEE Cat. No. 05CH37623C, (2005), p. 3952. 